\title{
PORTAFOLIOS DE ACTIVOS FINANCIEROS UTILIZANDO EL MODELO DE SHARPE Y TREYNOR
}

\author{
ASSETS PORTFOLIOS OF FINANCIAL \\ USING THE MODEL OF SHARPE AND TREYNOR \\ Nicko Alberto Gomero Gonzales* \\ Docente Principal de la Facultad de Ciencias Contables \\ Universidad Nacional Mayor de San Marcos-UNMSM / Lima-Perú \\ [Recepción: Marzo de 2014/ Conformidad: Mayo 2014]
}

\section{RESUMEN}

El mercado bursátil presenta muchas oportunidades de inversión, con expectativas de obtener buenos resultados que al final se puede traducir en rentabilidades sostenidas y crecientes para el inversionista, es más, si se opta por una buena estrategia, el riesgo asumido no podría comprometer la sostenibilidad del portafolio estructurado. Para este fin, las finanzas bursátiles presentan una serie de modelos que permite tomar una elección correcta cuando se demanda activos financieros; si bien en el grado de predictibilidad podría haber un sesgo entre lo que se observa y lo que se proyecta, pero no dejan de ser modelos interesantes para tomar decisiones en el mercado bursátil. El presente artículo, en su primera parte, se expone metodológicamente algunos de estos modelos, como el de Sharpe y de Treynor, que bien se podrían ajustar a los requerimientos de quienes están acostumbrados a gerenciar portafolios de activos financieros.

Palabras clave:

Mercado bursátil; riesgo; portafolios financieros; activos financieros.

\begin{abstract}
The stock market has many investment opportunities, with expectations of good results in the end will be translated into sustained and growing performance for the investor, if you choose a good strategy, the risk taken could not compromise the sustainability of structured portfolio. For that purpose, equity finance have a number of models that can make a right choice when demand financial assets; although the degree of predictability there might be a bias between what is observed and what is projected, but not without interest to make decisions in the stock market models. This article, in its first part, methodologically exposes some of these models, such as the Sharpe Ratio, which well could be adjusted to the requirements of those who are used to manage portfolios of financial assets.
\end{abstract}

Keywords:

Stock market; risk; financial portfolios; financial assets.

* Doctor en Ciencias Económicas. Magíster en Contabilidad con mención en Banca y Finanzas-UNMSM. Email: gomero_econ@yahoo.es 


\section{PRIMERA PARTE}

\section{INTRODUCCIÓN}

Si revisamos la literatura financiera nos podemos dar cuenta que existe toda una batería de modelos que al inversionista le conducirían a tomar la mejor decisión de inversión. Como es de entender, ni los especuladores entran a los mercados bajo la concepción de asumir mayor riesgo para ganar más. Esta aseveración que se escucha permanentemente en los foros académicos carece de todo sentido racional, si este principio fuera la filosofía que fundamentan los buenos resultados, solo bastaría estructurar carteras con activos altamente volatilizados ya que nos conducirían a obtener las más altas tasas de rentabilidad.

No hay que olvidar que el mercado es como el campo de batalla, quien gana una guerra no es aquel general que expone a sus soldados al fuego abierto del enemigo, sino, el éxito va a estar al lado de quien opte por la mejor estrategia, a lo mejor no es necesario ni atacar para asegurar el triunfo sino que bastaría con operaciones sicológicas para lograr el éxito. Como se puede apreciar, a la luz de esta estrategia el riesgo y los resultados favorables están inversamente correlacionados y muy cerca de cero.

Así es el mercado, no siempre gana el más vehemente o audaz, sino que el triunfo es para quien maneja mejor información, y plantea la mejor estrategia, para el mejor diseño financiero de predicción. En este sentido, en el mercado bursátil se puede ensayar una serie de estrategias de tal forma, que se pueda lograr buenos resultados pero sin exponerse en demasía a los riesgos. Para fundamentar lo señalado, solo basta ver la operatividad de la teoría de Markowitz, o algo más simple manejar matrices de correlaciones y covarianzas, o también la teoría del VAR (Valor en riesgo), el modelo de Sharpe, de Jensen, modelos que facilitan la toma de decisiones en el mercado de activos financieros.

El presente artículo estará basado en el desarrollo de algunos modelos financieros, a fin de quienes deseen tomar decisiones en el mercado bursátil lo hagan con mayores criterios, precisión y rendimiento.

\section{MARCO TEÓRICO}

\section{Modelo de Sharpe}

Una de las herramientas más usadas para seleccionar portafolio es el Índice de Sharpe, cuyo modelo contrasta el rendimiento promedio esperado del portafolio con el de un activo sin riesgo, considerándose comúnmente para este último caso el rendimiento de los bonos a 30 años que emite la Reserva Federal del Gobierno Norteamericano, que según los analistas sigue siendo considerado el activo con cero probabilidades de incumplimiento. Pero hay que tener presente que en la última crisis financiera internacional, la economía de Estados Unidos tuvo un fuerte debacle que comprometió las estructuras de su sistema financiero y de su economía real, depreciándose con ello sus activos de renta fija y variable; asimismo, el bono de la Reserva Federal. Por ello, es necesario que al manejar carteras los inversionistas busquen nuevas alternativas de tasa sin riesgo a fin de que el resultado obtenido sea más real y la decisión bursátil sea más acertada.

Cabe precisar, que el modelo no solo considera la prima de rendimiento, el cual es la diferencia entre al rendimiento promedio del portafolio y la tasa sin riesgo, sino que además, considera la volatilidad de la cartera, el cual es cuantificado por la desviación típica del portafolio. Este último indicador puede marcar la diferencia cuando se estructuraran carteras, por ejemplo si el inversionista tuviera como propósito obtener carteras poco riesgosas puede combinar activos cuyos coeficientes de correlación sea negativa y cercana a -1, esta elección conllevará a obtener un elevado índice de Sharpe. Los inversionistas abiertos al riesgo, tenderían a estructurar carteras con activos cuya correlación este cerca de 1 .

Teniendo este marco de referencia, es conveniente precisar que, al formarse dos carteras, se tendría que elegir aquella que posee mayor índice, para lograr este objetivo, se podría recomendar estructurar carteras con activos que estén inversamente correlacionados, ya que por este lado, se logrará obtener una menor desviación típica o riesgo para el portafolio, lo cual aumentaría la magnitud de su rendimiento. $\mathrm{O}$ en 
todo caso, adquirir activos que le generen una elevada prima por riesgo y a la vez, una baja desviación típica. Con esta estrategia de estructuración de carteras se estaría obteniendo una rentabilidad que aseguraría la ganancia esperada para el inversionista.

La fórmula que permite determinar el ratio de Sharpe es la siguiente:

$$
S h p=\frac{E(R)-R f}{\sigma p}
$$

Dónde:

Shp = Índice de Sharpe

$\mathrm{Ep}=$ rendimiento esperado del portafolio

$\mathrm{Rf}=$ tasa libre de riesgo

$\sigma \mathrm{p}=$ Riesgo del portafolio

La tasa de referencia representa la rentabilidad de un activo sin riesgo ( $\mathrm{Rf}$ ) que alternativamente al rendimiento del bono de la Reserva Federal, podría ser el rendimiento de un bono soberano con la mejor calificación de mercado, que es emitido por un gobierno.

Todo ratio de Sharpe negativo indica un rendimiento de la cartera inferior al de la rentabilidad del activo sin riesgo.

Todo ratio de Sharpe inferior a 1 indica una situación en la cual el rendimiento del activo es inferior al riesgo del mismo. Cuanto más alto sea el ratio, mejor.

\section{ÍNDICE DE JENSEN}

Permite evaluar el desempeño de un portafolio $y$ analizar de esta forma su rentabilidad en función del riesgo asumido. El cual es medido por el beta del activo o del portafolio.

La fórmula que permite determinar el índice de Jensen es como sigue:

$$
\text { lj }=E(R) p-[R f+B p(R m-R f)
$$

$\mathrm{E}(\mathrm{R}) \mathrm{p}=$ rendimiento del portafolio

$\mathrm{Rf}=$ tasa libre de riesgo

$\mathrm{Rm}=$ rendimiento promedio del mercado

Si el índice de Jensen es positivo, significa que el rendimiento observado promedio de la cartera será mayor que el rendimiento esperado teórico del mismo, lo cual sería conveniente para los inversionistas.
Si el índice de Jensen es negativo, implica que el rendimiento observado promedio de la cartera será menor que el rendimiento esperado teórico del portafolio, situación que conduciría a una situación de pérdida para el inversionista. En este caso, quien estructura el portafolio, por el riesgo asumido le exigirá un mayor retorno a los activos, pero no encontraría el retorno a esta exigencia de rentabilidad.

Una variable que juega un papel importante en este resultado es el beta del portafolio, encontrándose relacionado directamente con el riesgo de cada activo que conforma la cartera. Cuanto mayor sea este indicador, el inversionista le exigirá un elevado rendimiento teórico a su cartera, el cual deberá ser compensado con un mayor rendimiento promedio esperado del portafolio. Es decir, al escoger activos con altos betas o riesgos sistemáticos, exigiría obtener un elevado rendimiento promedio de la cartera, caso contrario, se obtendría un resultado diferente a lo que busca un inversionista.

Para lograr un elevado Índice de Jensen, situación que es favorable para el inversionista, la mejor alternativa, será conformar carteras con bajos betas y con elevados rendimientos promedios. Para la elección de activos con bajos betas, es necesario evaluar las covarianzas de los mismos contrastado con el rendimiento del mercado, no hay que perder de vista que a este último indicador se le tiene que determinar la varianza.

Le fórmula del beta se denota de la forma siguiente:

$$
\beta i=\frac{\operatorname{Cov}(R i, R m)}{\operatorname{Var}(R m)}
$$

Cov: Covarianza del rendimiento del activo y del mercado.

Var: Varianza del rendimiento del mercado.

En la fórmula se puede apreciar que, cuanto menor sea la covarianza del rendimiento del activo con relación al rendimiento del mercado, el beta será menor, lo cual induciría a obtener un resultado favorable para la cartera.

"El alfa de Jensen es el exceso de rendimiento del fondo con respecto al rendimiento de una cartera de mercado, con el mismo nivel de riesgo sistemático, luego de descontarles la tasa libre de riesgo que considera a los bonos del tesoro norteamerica- 
no. Ello con la finalidad de concentrar la evaluación financiera del portafolio en función al riesgo asumido por éste".

\section{RATIO DE TREYNor}

Mide el diferencial de rentabilidad obtenido sobre el activo libre de riesgo por unidad de riesgo sistemático o no diversificable del portafolio, representado por su Beta. Tomar el riesgo sistemático como medida de riesgo implica suponer que los inversionistas de carteras las administran de forma eficiente; es decir, que anulan el riesgo específico de los activos mediante la diversificación.

Cuanto mayor sea el ratio de Treynor mejor habrá sido la gestión de la cartera. Este indicador es una medida relativa que refleja la prima de rentabilidad de la cartera respecto al activo libre de riesgo por cada unidad de riesgo sistemático soportado por dicho portafolio.

$\mathrm{Al}$ apreciar la fórmula del índice se puede señalar que cuanto mayor es la prima de los rendimientos del portafolio y menor el riesgo sistemático, el cual está representado por el beta, mayor será el rendimiento de la cartera. Esta situación será favorable para el gestor de activos financieros de renta variable.

Tan igual como el índice de Jensen, para elegir carteras con bajos betas, es conveniente optar por activos que estén débilmente correlacionados con el mercado, pero que a la vez, tengan un alto rendimiento promedio esperado.

Hay que señalar que este modelo no toma en cuenta el riesgo único, porque una cartera bien diversificada, tiende a su eliminación. Por lo que, el único riesgo que interesa para gestionar carteras es el riesgo sistemático, es decir aquel que el inversionista no tiene opción de eliminar. Esta apreciación se visualiza en el siguiente gráfico:

\section{Gráfico $\mathrm{N}^{\circ} 1$ \\ Tipos de riesgos}

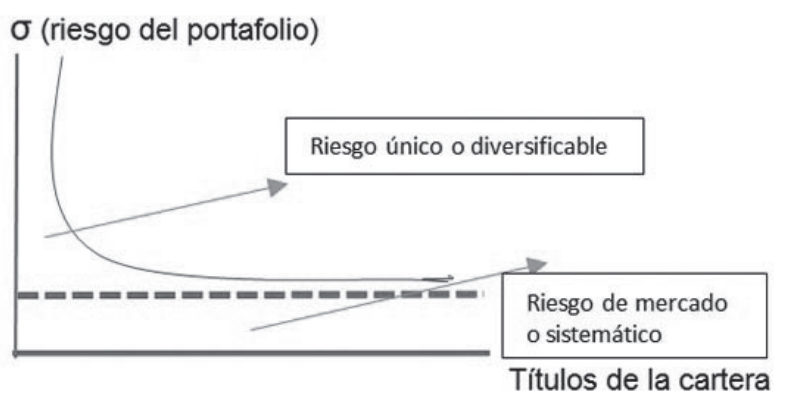

En el gráfico se puede visualizar que, el riesgo sistemático no cambia cuando el inversionista agrega títulos a su cartera, pero sucede lo contrario con el riesgo único, el cual tiende a eliminarse con la diversificación. Situación por la cual, una buena diversificación conducirá al gestor de carteras a enfrentar solo un tipo de riesgo que en este caso sería el de mercado, el cual, en el modelo de Treynor está representado por el beta de portafolio.

Para el ratio de Treynor se aplica la siguiente expresión matemática:

$$
R p=\frac{E(R) p-R f}{\beta p}
$$

Rp: Rentabilidad del portafolio.

Rf: Rentabilidad del activo sin riesgo (Tasa Libre de Riesgo)

$\beta \mathrm{p}$ : Beta de la cartera.

\section{APLICACIÓN METODOLÓGICA DE LOS MODELOS}

\section{RATIO DE SHARPE}

Para demostrar la operatividad del modelo de Sharpe, se ha considerado datos simulados para dos carteras. Cada una conformada por dos activos, cuyos rendimientos observados son para 8 meses. Hay que señalar que para obtener los rendimientos no se ha considerado los dividendos, solo se ha tomado como referencia la diferencia de precios de las acciones, para lo cual se tomó en cuenta la siguiente fórmula:

$$
\operatorname{Rit}=\left(\frac{\mathrm{Ri}_{t}}{\mathrm{Ri}_{\mathrm{t}-1}}-1\right) \times 100
$$


Dónde:

$\mathrm{Ri}_{\mathrm{t}}=$ rendimiento del activo en el periodo $\mathrm{t}$

$\mathrm{Ri}_{\mathrm{t}-1}=$ rendimiento del activo en el periodo anterior
A continuación se presenta los rendimientos de las acciones que conforman el primer portafolio.

Tabla $\mathbf{N}^{\circ} 1$

Rendimiento mensual de acciones ( \% )

(Primera cartera)

\begin{tabular}{|c|c|c|}
\hline PERIODO & RA & RB \\
\hline Enero & $10 \%$ & $7 \%$ \\
\hline Febrero & $12 \%$ & $9 \%$ \\
\hline Marzo & $15 \%$ & $14 \%$ \\
\hline Abril & $10 \%$ & $12 \%$ \\
\hline Mayo & $8 \%$ & $11 \%$ \\
\hline Junio & $10 \%$ & $13 \%$ \\
\hline Julio & $16 \%$ & $13 \%$ \\
\hline Agosto & $17 \%$ & $14 \%$ \\
\hline Setiembre & $15 \%$ & $10 \%$ \\
\hline
\end{tabular}

Fuente: Elaboración propia.

Gráfico $\mathrm{N}^{\circ} 2$

Rentabilidad de acciones de la primera cartera (\%)

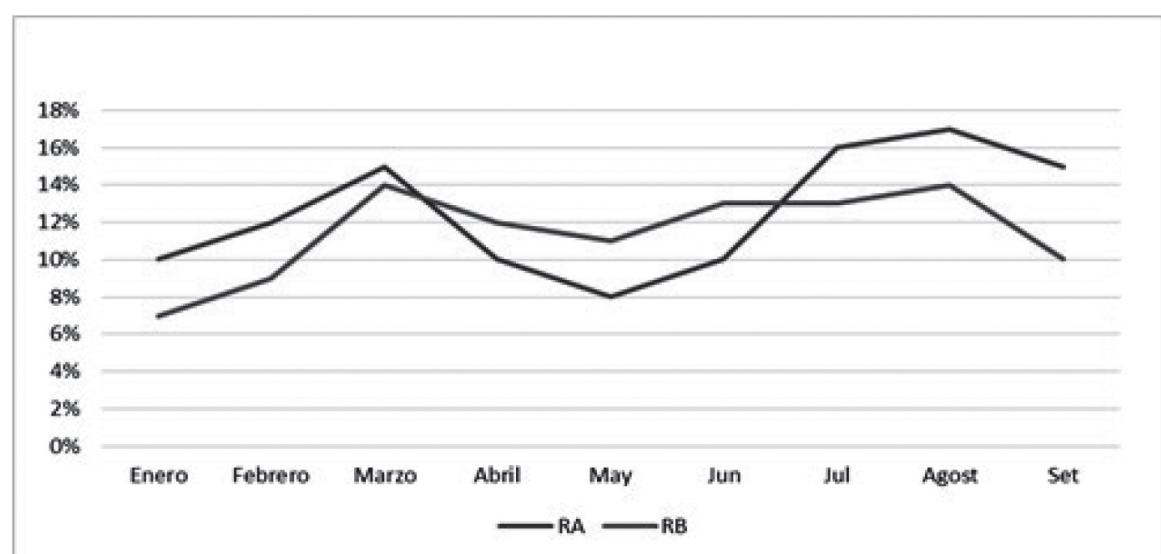

Fuente: Elaboración propia.

\section{Rendimiento promedio esperado de cada activo}

Para obtener el rendimiento promedio del portafolio se aplicó la fórmula siguiente:

$E(R) p=W A^{*} E(R) A+W B^{*} E(R) B$

Dónde:

$\mathrm{E}(\mathrm{R}) \mathrm{p}=$ Rendimiento del portafolio.

WA $=$ Participación del primer activo en el portafolio.

WB = Participación del segundo activo el portafolio.

$\mathrm{E}(\mathrm{R}) \mathrm{A}=$ Participación del primer activo en el portafolio.

$\mathrm{E}(\mathrm{R}) \mathrm{B}=$ participación del segundo activo en el portafolio.
Con una participación del $70 \%$ y $30 \%$ para cada activo respectivamente, se obtuvo un rendimiento promedio de la cartera del $12.22 \%$ mensual. Hay que señalar que el inversionista inclina su preferencia por el activo A, el cual presenta un mayor rendimiento promedio esperado. Por cada unidad monetaria de inversiones destina 70 centavos a esta acción y la diferencia le asigna al activo $\mathrm{B}$. 
Tabla $\mathbf{N}^{\circ} 2$

Rendimiento promedio de la cartera

\begin{tabular}{|c|c|c|c|}
\hline \multicolumn{4}{|c|}{ CARTERA 1 } \\
\hline Activo & $\mathbf{E}(\mathbf{R}) \mathbf{i}$ & $\begin{array}{c}\text { Participación } \\
\mathbf{\%}\end{array}$ & $\mathbf{E}(\mathbf{R}) \mathbf{p}$ \\
\hline A & $13 \%$ & 0.7 & $8.79 \%$ \\
\hline B & $11 \%$ & 0.3 & $3.43 \%$ \\
\hline E ( R $) \mathrm{p}$ & & & $12.22 \%$ \\
\hline
\end{tabular}

\section{Riesgo de los activos conformantes de la cartera}

El activo A si bien presenta mayor rendimiento promedio pero a la vez, refleja un mayor riesgo, el cual se aprecia en el grado de la desviación típica, el cual llega al 3.06\%, ubicándose por encima del activo B, cuya volatilidad solo llega al $2.27 \%$. Entre ambos activos existe una correlación directa, pero no tan fuerte, ya que el coeficiente de asociación solo llega al $46.12 \%$.

Tabla $\mathbf{N}^{\circ} 3$

Desviación típica de cada activo y coeficiente de correlación de los activos de la primera cartera

\begin{tabular}{|c|c|c|}
\hline & A & B \\
\hline Des. Típica & $3.06 \%$ & $2.27 \%$ \\
\hline Correlación & $46.12 \%$ & \\
\hline
\end{tabular}

Por el resultado de la desviación típica se aprecia que el activo A es más sensible o riesgoso que el activo B. Es conveniente precisar, que para determinar el ratio de Sharpe se necesita obtener el riesgo de la cartera y no solamente de los activos en forma separa. El riesgo de la cartera, se cuantifica a través de la desviación típica del mismo.

\section{Riesgo de la cartera}

Hay que recordar que el modelo de Sharpe, exige determinar el riesgo del portafolio, el cual es medido por la desviación típica de la cartera compuesta por dos activos. Los estadísticos básicos para determinar este tipo de riesgo son: la participación de cada activo en la cartera $(\mathrm{Wi})$, varianza de los activos ( $\sigma \mathrm{i})$ y su grado de correlación.
Tabla $\mathrm{N}^{\circ} 4$

Varianza y desviación típica de la cartera

\begin{tabular}{|l|l|}
\hline Varianza & $0.06 \%$ \\
\hline D. típica & $2.53 \%$ \\
\hline
\end{tabular}

Para determinar la desviación típica de la cartera se aplicó la fórmula que sigue

Varianza:

$\sigma^{\wedge} 2: \mathrm{D} 19^{\wedge} 2^{*} \mathrm{C} 13^{\wedge} 2+\mathrm{D} 20^{\wedge} 2^{*} \mathrm{D} 13^{\wedge} 2+2^{*}\left(\mathrm{D} 19^{*} \mathrm{D} 20^{*} \mathrm{C} 14^{*} \mathrm{C} 13^{*} \mathrm{D} 13\right)$

D. típica;

op: $\sqrt{\mathrm{D} 19^{\wedge} 2^{*} \mathrm{C} 13^{\wedge} 2+\mathrm{D} 20^{\wedge} 2^{*} \mathrm{D} 13^{\wedge} 2+2^{*}\left(\mathrm{D} 19^{*} \mathrm{D} 20^{*} \mathrm{C} 14^{*} \mathrm{C} 13^{*} \mathrm{D} 13\right)}$

\section{Ratio de Sharpe de la primera cartera}

Con los datos obtenidos, se determinó el ratio de Sharpe, el cual llega a 3.25.

$$
\mathrm{ISH}=\frac{(12.22 \%-4 \%)}{2.53 \%}
$$

ISH $=\mathbf{3 . 2 5}$

Tabla $\mathbf{N}^{\circ} 5$

Rendimiento mensual de acciones (\%) (Segunda cartera)

\begin{tabular}{|c|c|c|}
\hline PERIODO & RC & RD \\
\hline Enero & $14 \%$ & $5 \%$ \\
\hline Febrero & $8 \%$ & $10 \%$ \\
\hline Marzo & $16 \%$ & $7 \%$ \\
\hline Abril & $8 \%$ & $12 \%$ \\
\hline Mayo & $13 \%$ & $8 \%$ \\
\hline Junio & $7 \%$ & $11 \%$ \\
\hline Julio & $11 \%$ & $9 \%$ \\
\hline Agosto & $6 \%$ & $15 \%$ \\
\hline Setiembre & $6 \%$ & $18 \%$ \\
\hline
\end{tabular}

Con la rentabilidad observada para los activos de la segunda cartera (C y D), se sigue el mismo procedimiento seguido para el primer portafolio para determinar el ratio de Sharpe. 


\section{Gráfico $\mathrm{N}^{\circ} 3$}

Rentabilidad de acciones de la segunda cartera (\%)

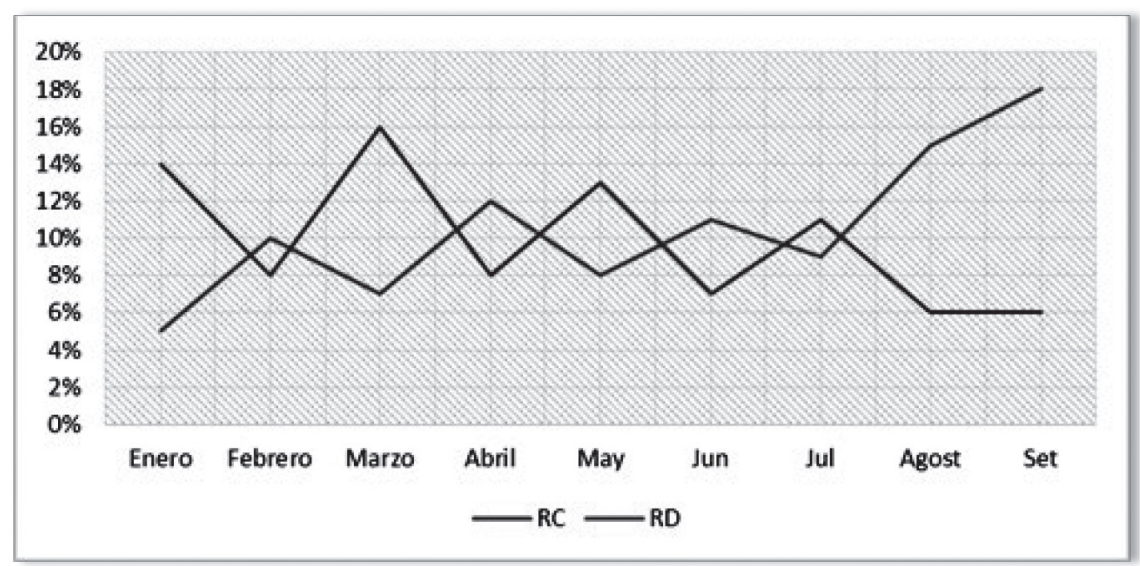

Fuente: Elaboración propia.

\section{Rendimiento promedio esperado de cada activo}

El inversionista asigna un $70 \%$ de su riqueza al primer activo, cuyo rendimiento promedio es menor que el activo $\mathrm{D}$. el rendimiento esperado promedio del portafolio es del 10,09\%.

Tabla $\mathbf{N}^{\circ} 6$

\section{Desviación típica de cada activo y coeficiente de correlación}

\begin{tabular}{|c|c|c|c|}
\hline Activo & $\mathbf{E}(\mathbf{R}) \mathbf{i}$ & $\begin{array}{c}\text { Participación } \\
\mathbf{\%}\end{array}$ & $\mathbf{E}(\mathbf{R}) \mathbf{p}$ \\
\hline C & $10 \%$ & 0.7 & $6.92 \%$ \\
\hline D & $11 \%$ & 0.3 & $3.17 \%$ \\
\hline TOTAL & & & $\mathbf{1 0 . 0 9 \%}$ \\
\hline
\end{tabular}

Riesgo de los activos conformantes de la cartera

Ambos activos no presentan mucha diferencia en su volatilidad. Lo importante es que en este caso, el inversionista opta por la cobertura ya que estructura con activos inversamente correlacionados, posición que influenciará en el resultado del riesgo del portafolio.

\section{Tabla $\mathbf{N}^{\circ} 7$}

Desviación típica de cada activo y coeficiente de correlación de los activos de la primera cartera

\begin{tabular}{|c|c|c|}
\hline & C & D \\
\hline Des. Típica & $3.51 \%$ & $4.80 \%$ \\
\hline Correlación & $-86 \%$ & \\
\hline
\end{tabular}

\section{Riesgo de la cartera}

Por el resultado del coeficiente de correlación, se evidencia que los activos conformantes de la cartera están inversamente relacionados, lo cual proyecta una situación de cobertura para el inversionista. No hay que olvidar que, cuando un portafolio está conformado por activos con una correlación de -1 , se dice que el inversionista está perfectamente con cobertura ante riesgos, situación que no se presenta en este caso, ya que el coeficiente correlación llega al - $86 \%$, pero si se puede argumentar que es un inversionista adverso al riesgo. Esta combinación de activos al conllevar a una menor desviación típica del portafolio, influenciará positivamente en el ratio de Sharpe.

Tabla $\mathbf{N}^{\circ} 8$

\section{Varianza y desviación típica de la cartera}

\begin{tabular}{|l|l|}
\hline Varianza & $0.03 \%$ \\
\hline D. típica & $1.58 \%$ \\
\hline
\end{tabular}

Ratio de Sharpe de la segunda cartera

$$
\mathrm{ISH}=\frac{(10.09 \%-4 \%)}{1.58 \%}
$$

$$
\text { ISH }=\mathbf{3 . 8 4}
$$

\section{Toma de decisiones}

Al comparar las dos carteras, se llega a establecer que la segunda cartera sería más rentable para el inversionista. La variable crítica que influyó en 
este resultado fue el coeficiente de correlación y por ende, la desviación típica de los activos. La segunda cartera está conformada por activos con cobertura (correlación negativa) en contraste con el primer portafolio, cuyos rendimientos de los activos están directamente relacionados, por ello es que el coeficiente de correlaciones es positivo e influye a que el riesgo de la cartera sea mayor, lo cual induce a obtener un menor coeficiente de Sharpe. Eligiendo la segunda cartera el inversionista estaría en posición financiera.

\section{RATIO DE Treynor}

Para determinar este índice de Treynor se tomará en cuenta 4 activos $(1,2,3,4)$ y a diferencia al modelo de Sharpe se introducirá el rendimiento del mercado a fin de determinar el beta de los activos y con ello el del portafolio.
Tabla $\mathbf{N}^{\circ} 9$

\section{Cartera 1}

\begin{tabular}{|c|c|c|c|}
\hline MES & RM & R1 & R2 \\
\hline 1 & $18 \%$ & $12 \%$ & $20 \%$ \\
\hline 2 & $15 \%$ & $8 \%$ & $8 \%$ \\
\hline 3 & $12 \%$ & $7 \%$ & $-2 \%$ \\
\hline 4 & $13 \%$ & $6 \%$ & $8 \%$ \\
\hline 5 & $17 \%$ & $10 \%$ & $12 \%$ \\
\hline 6 & $20 \%$ & $15 \%$ & $17 \%$ \\
\hline 7 & $22 \%$ & $17 \%$ & $18 \%$ \\
\hline 8 & $18 \%$ & $15 \%$ & $7 \%$ \\
\hline 9 & $15 \%$ & $10 \%$ & $5 \%$ \\
\hline 10 & $12 \%$ & $9 \%$ & $-2 \%$ \\
\hline
\end{tabular}

Fuente: Elaboración propia.

\section{Gráfico $\mathrm{N}^{\circ} 4$}

\section{Rendimiento de los activos 1 y 2 y del mercado de valores de la primera cartera (\%)}

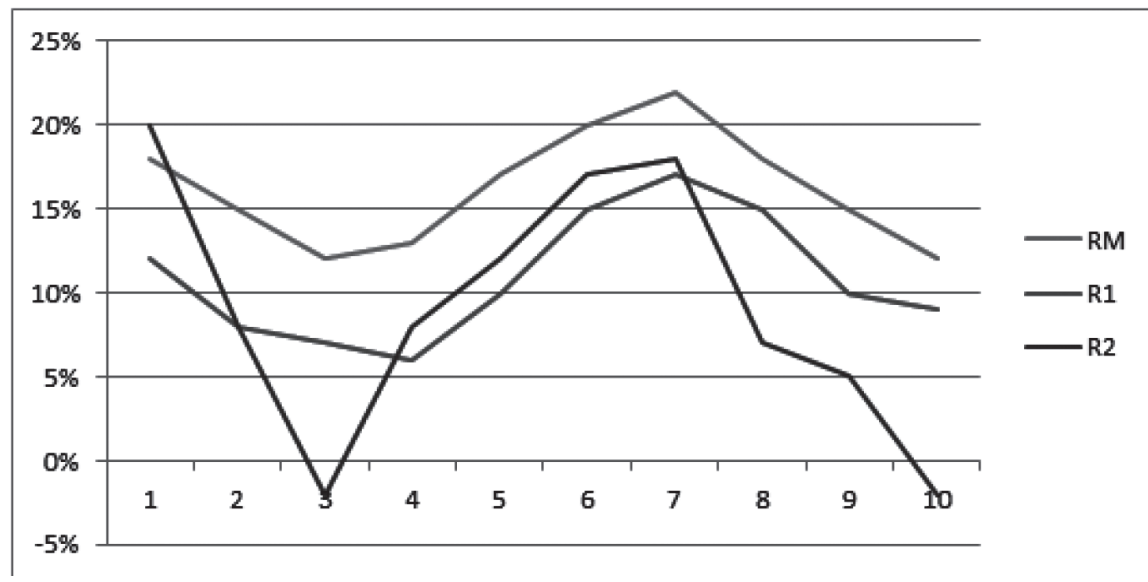

Fuente: Elaboración propia.

A continuación se determina el beta de cada activo, para ello se ha utilizado el método de regresión lineal simple, en cuya ecuación encontramos dos parámetros, el $\beta 1$ y $\beta 2$. Siendo el segundo ( $\beta 2)$ el beta de cada activo, el cual representa además, la pendiente de la línea de regresión. La ecuación general es como sigue:

$$
\begin{aligned}
& \mathrm{Yi}=\beta 1+\beta 2(\mathrm{Xi}) \quad 0 \\
& \mathrm{Ri}=\beta 1+\beta 2(\mathrm{Rm})
\end{aligned}
$$

Siendo:

$\beta 2=$ beta del activo $\mathrm{Rm}=$ rendimiento del mercado

\section{Determinando el beta de los activos de la primera cartera}

Como ya se mencionó, para definir el beta del activo se determinará la línea de regresión y con ello, su ecuación respectiva. La pendiente de esta línea mide el beta del activo. En el caso presentado, el primer activo posee un beta de 1.00, por lo que se puede argumentar, que su riesgo es igual al del mercado. El resultado del beta también se puede utilizar la siguiente fórmula:

$$
\mathrm{B} 1=\frac{\operatorname{Cov}(\mathrm{Rm}, \mathrm{R} 1)}{\operatorname{Var}(\mathrm{Rm})}
$$


El resultado aplicando esta fórmula debe coincidir con el de la pendiente de la línea de regresión lineal.

Gráfico $\mathbf{N}^{\circ} 5$

Línea y ecuación de regresión para el primer activo

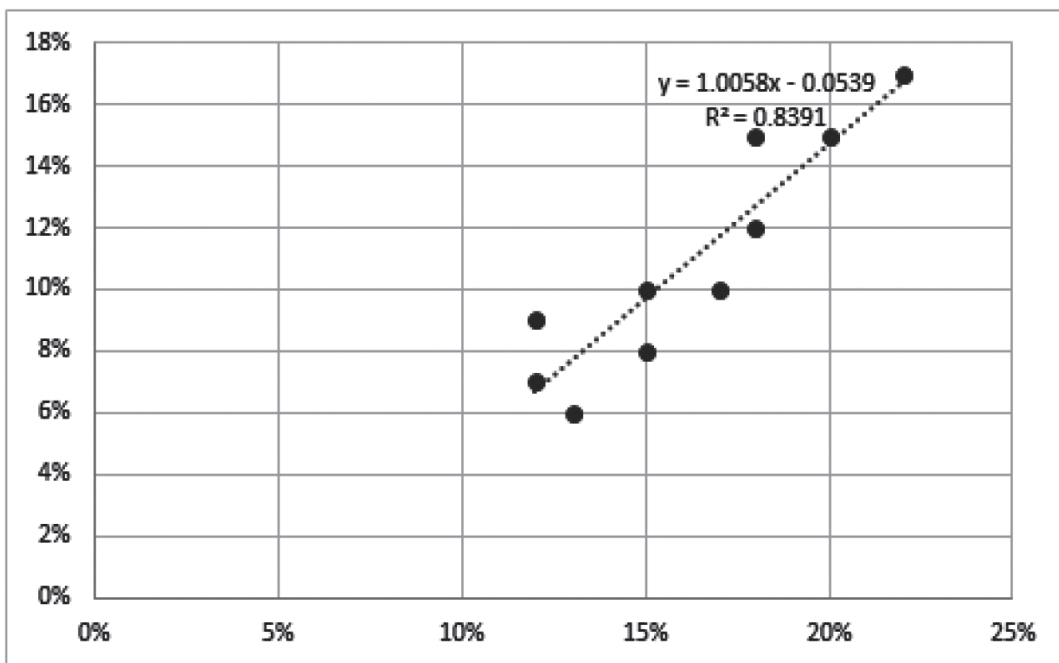

Fuente: Elaboración propia.

A continuación, siguiendo el mismo procedimiento se procederá a obtener el beta del segundo activo. Se puede apreciar en el gráfico $\mathrm{N}^{\circ} 6$ que la línea de regresión de este segundo activo es más incli- nada que el primero, por lo que conducirá a obtener un mayor beta. Es así, al determinar la ecuación de regresión se llegó a obtener un beta de 1.96 .

Gráfico $\mathrm{N}^{\circ} 6$

Línea y ecuación de regresión para el segundo activo

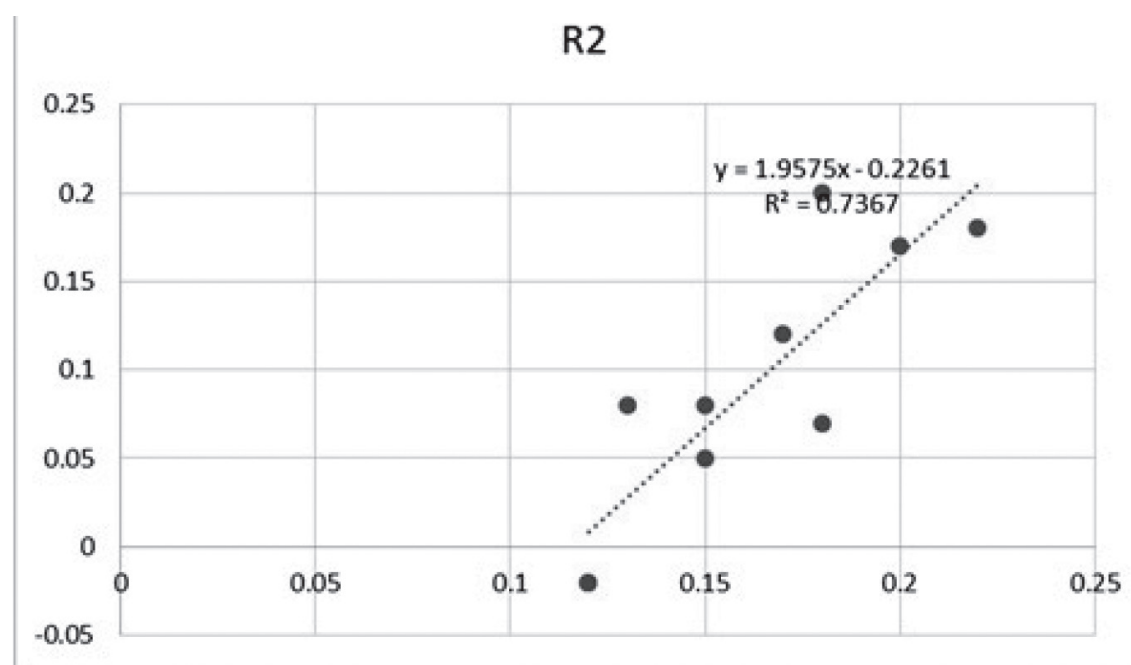

Fuente: Elaboración propia.

Como se señaló el beta de este activo es de 1.96. Como el resultado es mayor a 1 , se puede calificar como un activo de alto riesgo. Este activo presenta más volatilidad que el mercado. Comparativamente, el activo 2 con respecto al 1 se califica como de mayor riesgo o volatilidad. 
Tabla $\mathrm{N}^{\circ} 10$

Rendimiento de los activos y del mercado de la segunda cartera

\begin{tabular}{|c|c|c|c|}
\hline MES & $\mathbf{R M}$ & $\mathbf{R 2}$ & $\mathbf{R 3}$ \\
\hline 1 & $18 \%$ & $10 \%$ & $8 \%$ \\
\hline 2 & $15 \%$ & $10 \%$ & $7 \%$ \\
\hline 3 & $12 \%$ & $0 \%$ & $6 \%$ \\
\hline 4 & $13 \%$ & $8 \%$ & $8 \%$ \\
\hline 5 & $17 \%$ & $11 \%$ & $11 \%$ \\
\hline 6 & $20 \%$ & $11 \%$ & $16 \%$ \\
\hline 7 & $22 \%$ & $13 \%$ & $16 \%$ \\
\hline 8 & $18 \%$ & $11 \%$ & $12 \%$ \\
\hline 9 & $15 \%$ & $10 \%$ & $9 \%$ \\
\hline 10 & $12 \%$ & $10 \%$ & $7 \%$ \\
\hline
\end{tabular}

Fuente: Elaboración propia.

$\mathrm{R} 2$

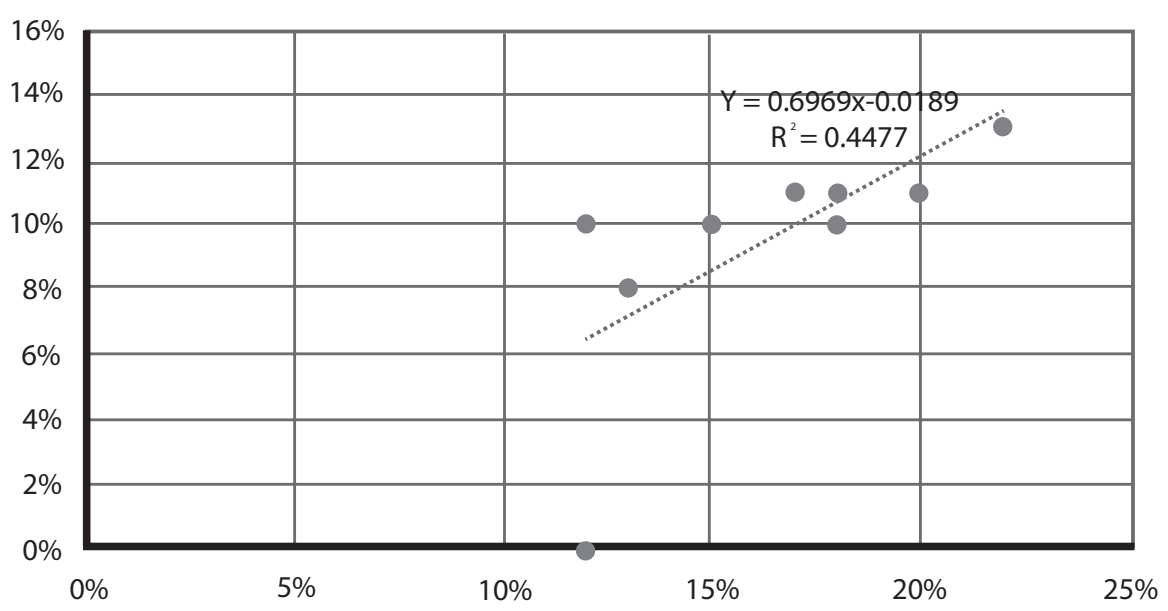

Fuente: Elaboración propia.

R3

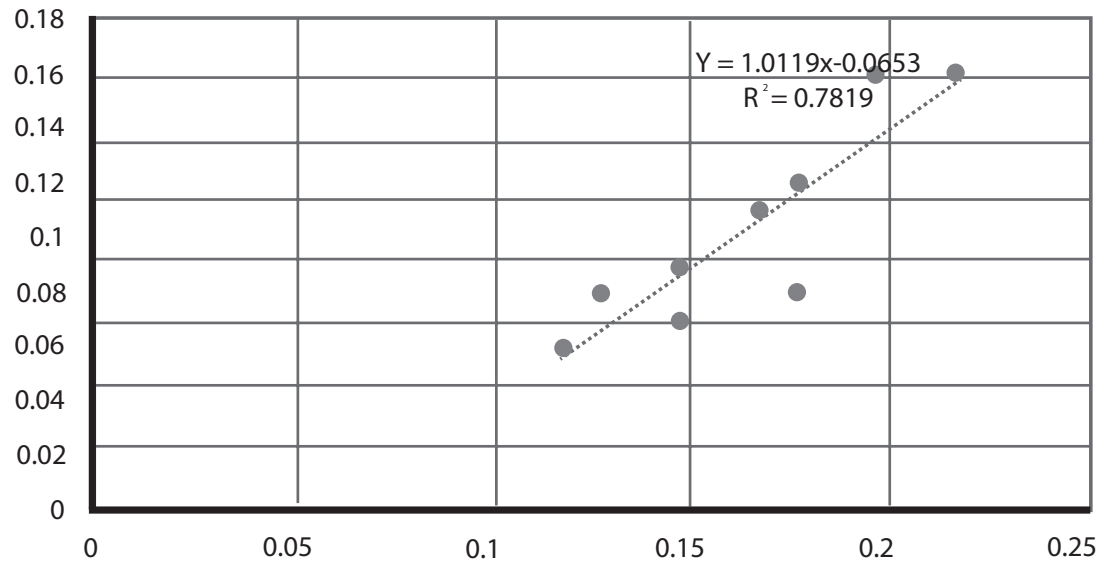

Fuente: Elaboración propia.

144/ QVIPURAMAYOC | Vol. 22(41) 2014 
Los dos activos de esta cartera a diferencia de la primera presentan un bajo riesgo. El activo 2 es menos volátil que el mercado. En cuanto al primer activo se puede señalar que, si el mercado avanza en su rendimiento en $1 \%$, este activo crecerá solo en un $0.70 \%$. Dado estos resultados, el beta de este portafolio será menor que el beta de la primera cartera. Resultados que marcarán la diferencia en el ratio de Treynor. A continuación se determinan el beta de ambas carteras:

\section{Beta del portafolio}

Portafolio 1

\begin{tabular}{|c|c|c|c|}
\hline & $\mathrm{Bi}$ & $\%$ & $\mathrm{Bp}$ \\
\hline 1 & 1 & 0.3 & 0.3 \\
\hline 2 & 1.96 & 0.7 & 1.372 \\
\hline & & & 1.672 \\
\hline
\end{tabular}

\section{Portafolio 2}

\begin{tabular}{|c|c|c|c|}
\hline & $\mathrm{Bi}$ & $\%$ & $\mathrm{Bp}$ \\
\hline 1 & 0.7 & 0.3 & 0.21 \\
\hline 2 & 1.01 & 0.7 & 0.707 \\
\hline & & & 0.917 \\
\hline
\end{tabular}

El portafolio 1 es más riesgoso que el portafolio 2. Si el mercado avanza en $1 \%$, con la primera cartera se ganara $1.67 \%$, con la segunda de obtendrá $0.91 \%$. Pero si el mercado retrocede, con la primera cartera se obtendrá mayores pérdidas. En sí, la primera cartera avanza más rápidamente que el mercado, por ello se le califica como de alto riesgo. En ambos casos el inversionista le da mayor preferencia al activo 2, asignándole un $70 \%$ de sus presupuesto.

\section{Rendimientos promedios de la cartera}

Rendimiento promedio de la cartera 1

\begin{tabular}{|c|c|c|c|}
\hline & $\mathrm{E}(\mathrm{R}) \mathrm{i}$ & $\%$ & $\mathrm{E}(\mathrm{Rp})$ \\
\hline 1 & $10.90 \%$ & 0.3 & 0.0327 \\
\hline 2 & $9.10 \%$ & 0.7 & 0.0637 \\
\hline & & & 0.0964 \\
\hline
\end{tabular}

Rendimiento promedio de la cartera 2

\begin{tabular}{|c|c|c|c|}
\hline & $\mathrm{E}(\mathrm{R}) \mathrm{i}$ & $\%$ & $\mathrm{E}(\mathrm{R}) \mathrm{p}$ \\
\hline 1 & $9.40 \%$ & 0.3 & 0.0282 \\
\hline 2 & $10.00 \%$ & 0.7 & 0.07 \\
\hline & & & 0.0982 \\
\hline
\end{tabular}

Al comparar ambas carteras, se puede observar, que la segunda cartera presenta un mayor rendimiento promedio esperado.

$\mathrm{Al}$ suponer que la tasa sin riesgo es del 4\% (Rf), el coeficiente de Treynor, será igual a:

Primera cartera:

$$
\begin{aligned}
& \mathrm{Rp}=\frac{\mathrm{E}(\mathrm{R}) \mathrm{p}-\mathrm{Rf}}{\beta \mathrm{p}} \\
& \mathrm{Rp} 1=\frac{9.64 \%-4 \%}{1.67}=0.034
\end{aligned}
$$

Segunda cartera:

$$
\mathrm{Rp} 2=\frac{9.82 \%-4 \%}{0.917}=0.063
$$

Al comparar los resultados se puede apreciar que la cartera 2 es más rentable que la cartera 1 . El indicador que marca la diferencia en este resultado es el beta de ambos activos.

\section{CONCLUSIONES}

1. El ratio de Sharpe y de Treynor son indicadores de rentabilidad que permiten tomar decisiones para elegir las mejores carteras de activos de renta variable.

2. Es conveniente elegir carteras con activos cuyas covarianzas sean negativas, de esta forma se podrá minimizar el riesgo del portafolio, el cual se ha de reflejar en el resultado de la desviación típica. Con esta elección se podrá lograr estructurar carteras con elevados ratios de Sharpe.

3. Los betas de las carteras influyen en los resultados del índice de Treynor, se recomienda elegir activos de bajos betas o riesgo sistemático con elevados riesgos promedios esperados. De esta forma, se podrá conseguir una elevada rentabilidad para el portafolio. 
4. Los modelos de cartera, al incluir una serie de variables estadísticas que proyectan riesgos y rendimientos, permiten optimizar decisiones en el mercado especulativo, como lo es el bursátil.

\section{REFERENCIAS BIBLIOGRÁFICAS}

1. BODIE, ZVI y MERTON, ROBERT (1999). “Finanzas”. México: Prentice Hall. Capítulo 10 (págs. 215-242).

2. MASCAREÑAS, JUAN (2008) "El coste del capital”. Universidad Complutense de Madrid. España. [electrónico www.econfinanzas.com]
3. Pérez-Carballo, A., Vela Sastre, E. (1997) Principios de gestión financiera de la empresa, Alianza, Madri.

4. Purcell, W. R. Jr. (1981), Cómo comprender las finanzas de una compañía, Norma.

5. Durán Herrera, Juan José (1992). “Economía y Dirección Financiera de la Empresa” Editorial Piramide. 